\title{
An asymptomatic case of peritoneal encapsulation: case report and review of the literature
}

\author{
Omer S Al-Taan, Martyn D Evans', Javid A Shami
}

\begin{abstract}
Peritoneal encapsulation is a rare congenital anomaly characterised by a thin membrane of peritoneum encasing the small bowel to form an accessory peritoneal sac. We present a case of peritoneal encapsulation diagnosed incidentally in an 82 year old man undergoing laparotomy for colonic cancer. The sac was easily excised and surgery was otherwise uneventful. A discussion of the case and a review of the literature are presented.
\end{abstract}

\section{Introduction}

Peritoneal encapsulation (PE) is a very rare condition that is characterised by a thin membrane encasing the small bowel forming an accessory peritoneal sac. Cases usually present with small bowel obstruction or can be an incidental finding during laparotomy. We present a case of PE diagnosed during surgery for a colon cancer.

\section{Case report}

An 82 year old white male presented to the out patient clinic complaining of left iliac fossa pain and was diagnosed with a descending colon cancer. He had never undergone abdominal surgery previously. The tumour was thought to be at risk of obstructing the colon and a potentially curative surgery was planned. During operation and after entering the peritoneal cavity the small bowel was entirely covered with a thin membrane that had the appearance of an accessory peritoneum through which the small bowels were visible (see figure 1, figure 2 , figure 3 and figure 4). Excision of the membrane released the small bowels. There was no associated small bowel pathology or adhesions. A palliative Hartmann's procedure was performed as peritoneal metastases were encountered during surgery. The patient made a good recovery post operatively and was discharged from hospital after five days.

\footnotetext{
* Correspondence: emarteva@hotmail.com
General Surgery Department, Princess of Wales Hospital, (Coity Road),

* Correspondence: emarteva@hotmail.com
General Surgery Department, Princess of Wales Hospital, (Coity Road), Bridgend, (CF31 1PU), UK
}

(C) 2010 Al-Taan et al; licensee BioMed Central Ltd. This is an Open Access article distributed under the terms of the Creative Commons Attribution License (http://creativecommons.org/licenses/by/2.0), which permits unrestricted use, distribution, and reproduction in any medium, provided the original work is properly cited.

\section{Discussion}

Peritoneal encapsulation is a very rare condition characterised by what looks to be an accessory peritoneal membrane covering all or part of the small bowels [1]. This is attached to the ascending and descending colon laterally, the transverse colon superiorly and the posterior surface of the parietal peritoneum inferiorly. It is believed to be caused by mal-rotation of the bowel during the $12^{\text {th }}$ week of gestation [1], this causes the formation of an accessory sac from the peritoneum covering the umbilicus. The membrane may cover the entire or part of the small bowel from the duodeno-jejunal junction down to the ileo-colic junction [2].

The two commonest clinical presentations are: acute small bowel obstruction or incidental diagnosis during laparotomy for another condition [4], however, many cases are incidental findings at autopsy. Some patients may have episodes of intermittent colicky abdominal pain or episodes or sub-acute small bowel obstruction, prior to a definitive diagnosis,

Diagnosis of PE pre-operatively may be impossible because plain abdominal $\mathrm{x}$-ray may be normal or only show dilated small bowel loop and CT scan findings can be very non-specific [3]. Membrane division during surgery is curative (assuming there is no ischaemic bowel) [2]. On literature search no re-operation was reported on PE after dividing the encasing membrane.

Knowledge of this congenital anomaly is of potential use to the abdominal surgeon, as its presence is not reported in standard anatomical descriptions. Its presence is a source of potential confusion, particularly in 


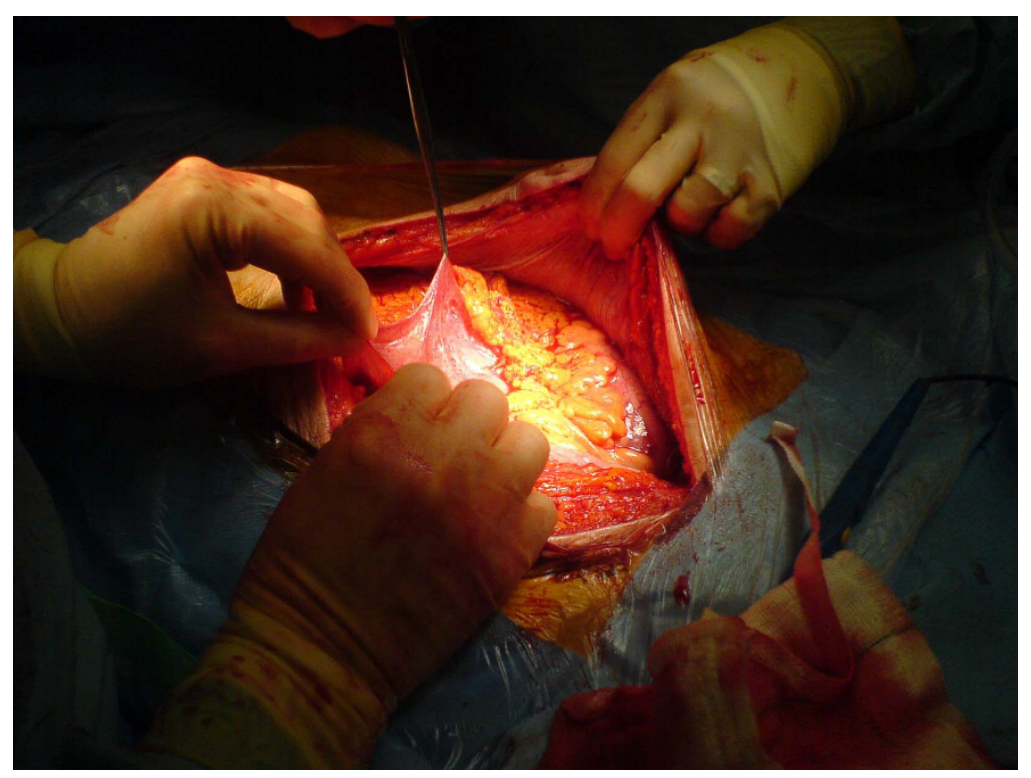

Figure 1 Peritoneal encaspulation of the small bowel observed after opening the abdomen.

laparoscopic surgery after establishing pneumo peritoneum. The use of laparoscopy to carry out abdominal surgery is increasing, knowledge of this anatomical anomaly would allow a surgeon, who recognised the membrane as $\mathrm{PE}$, to divide the membrane laparoscopically without recourse to conversion to open laparotomy.

\section{Consent}

Written informed consent was obtained from the patient for publication of this case report and accompanying images. A copy of the written consent is available for review by the Editor-in-Chief of this journal.

\section{Authors' contributions}

OA prepared the manuscript. ME carried out the literature search and edited the manuscript. JS conceived the idea to write the case report and edited the manuscript. All authors read and approved the manuscript.

\section{Competing interests}

The authors declare that they have no competing interests.

\section{Received: 14 October 2009}

Accepted: 9 January 2010 Published: 9 January 2010

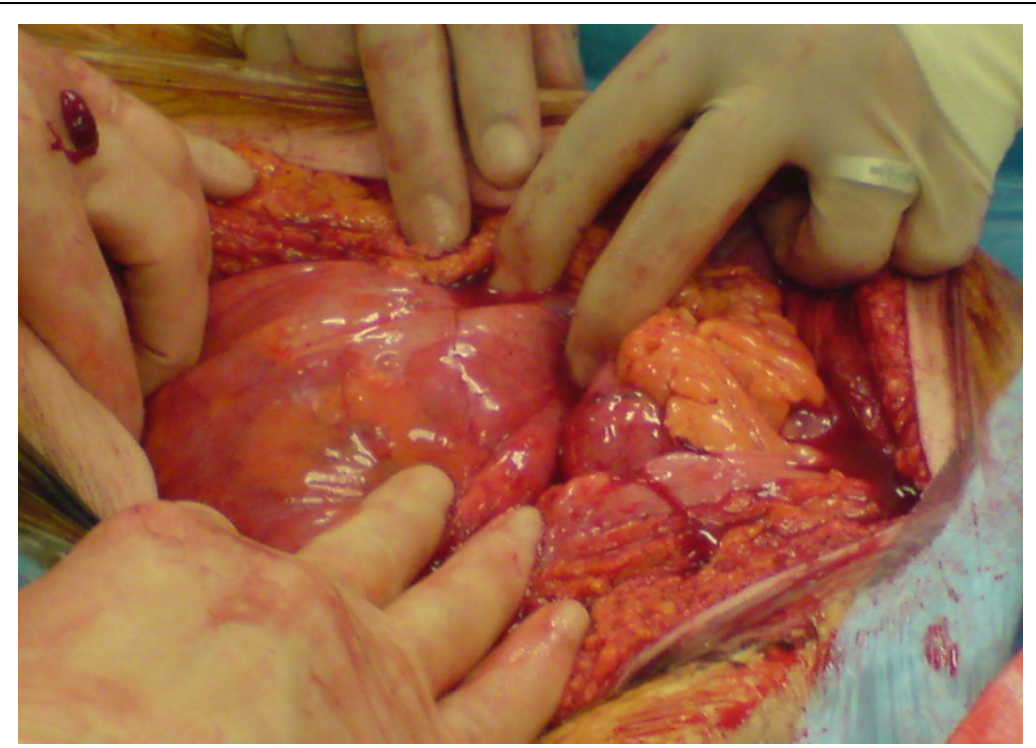

Figure 2 Small bowel loops visible through the sheet of the peritoneal encapsulation. 


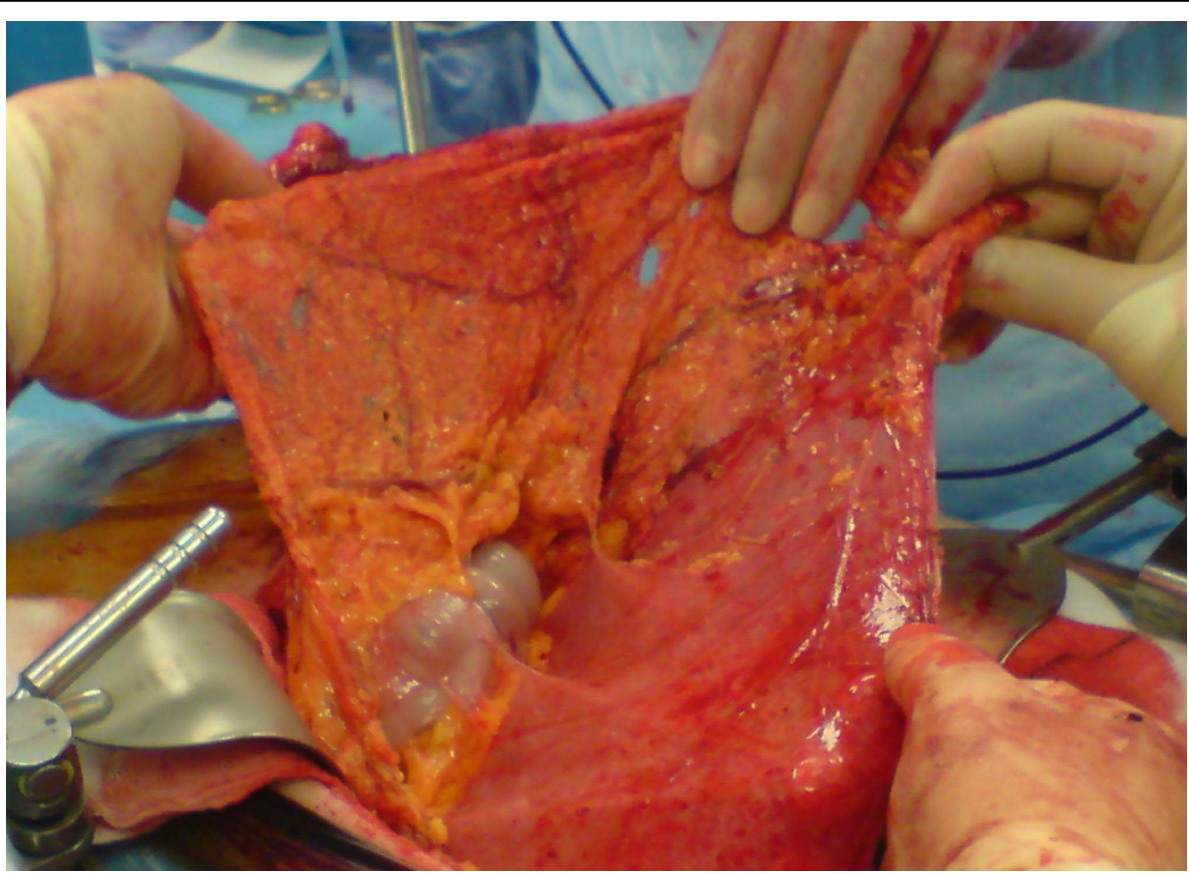

Figure 3 Superior attachment of PE to the inferior surface of the transverse colon with greater omentum.above, inferior and lateral attachments have been divided.

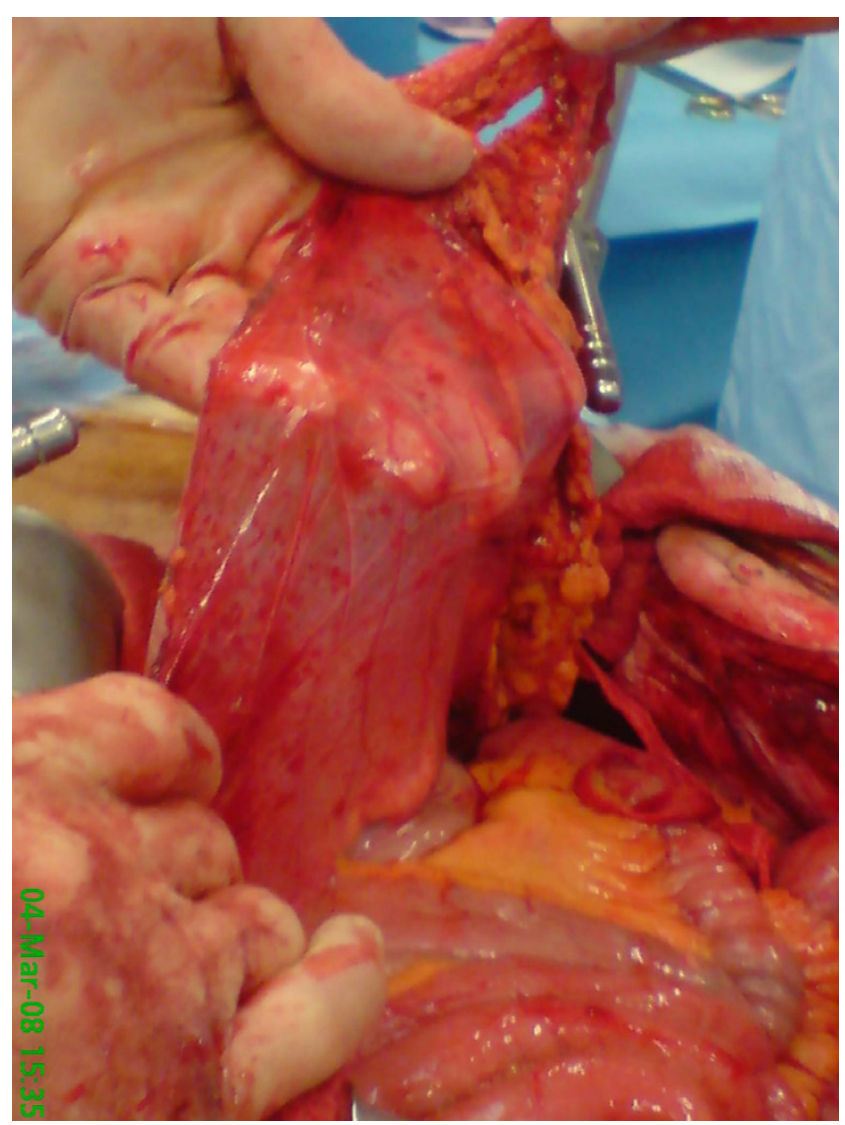

Figure 4 Membrane of peritoneal encapsulation is being held up by the operating surgeon, with the small bowel visible inferiorly. 


\section{References}

1. Naraynsingh V, Maharaj D, Singh M, Ramdass MJ: Peritoneal encapsulation: a preoperative diagnosis is possible. Postgrad Med J 2001, 77:725-726.

2. Sherigar* JM, McFall B, Wali J: Peritoneal encapsulation: presenting as small bowel obstruction in an elderly woman. Ulster Med J 2007, 76(1):4244.

3. Chew MH, Sophian Hadi I, Chan G, Ong HS, Wong WK: A problem encapsulated: the rare peritoneal encapsulation syndrome. Singapore Med J 2006, 47(9):808-10.

4. Wirnsberger GH, Ganser K, Domej W, Sauseng G, Moore D, Moczygemba M, Krejs GJ: Sclerosing encapsulating peritonitis: differential diagnosis to peritoneal encapsulation and abdominal cocoon. Z Gastroenterol 1992, 30(8):534-7.

doi:10.1186/1757-1626-3-13

Cite this article as: Al-Taan et al:: An asymptomatic case of peritoneal encapsulation: case report and review of the literature. Cases Journal 2010 3:13.

Publish with Biomed Central and every scientist can read your work free of charge

"BioMed Central will be the most significant development for disseminating the results of biomedical research in our lifetime. "

Sir Paul Nurse, Cancer Research UK

Your research papers will be:

- available free of charge to the entire biomedical community

- peer reviewed and published immediately upon acceptance

- cited in PubMed and archived on PubMed Central

- yours - you keep the copyright 